Research Paper

\title{
The Combination Of Weak Expression Of PRDX4 And Very High MIB-1 Labelling Index Independently Predicts Shorter Disease-free Survival In Stage I Lung Adenocarcinoma
}

\author{
Akihiro Shioya ${ }^{1}$, Xin Guo ${ }^{1}$, Nozomu Motono ${ }^{2}$, Seiya Mizuguchi ${ }^{3}$, Nozomu Kurose ${ }^{1,3}$, Satoko Nakada ${ }^{1,3}$, \\ Akane Aikawa1,3, Yoshitaka Ikeda ${ }^{4}$, Hidetaka Uramoto² ${ }^{2}$ Sohsuke Yamada ${ }^{1,3 凶}$ \\ 1. Department of Pathology and Laboratory Medicine, Kanazawa Medical University, Ishikawa. \\ 2. Department of Thoracic Surgery, Kanazawa Medical University, Ishikawa. \\ 3. Department of Pathology, Kanazawa Medical University Hospital, Ishikawa. \\ 4. Division of Molecular Cell Biology, Department of Biomolecular Sciences, Saga University Faculty of Medicine, Saga, Japan. \\ $\triangle$ Corresponding author: Sohsuke Yamada, M.D., Ph.D., Department of Pathology and Laboratory Medicine, Kanazawa Medical University, 1-1 Daigaku, \\ Uchinada, Ishikawa, 920-0293, Japan. Tel: 81-76-218-8264; Fax: 81-76-286-1207; and E-mail: sohsuke@kanazawa-med.ac.jp \\ (C) Ivyspring International Publisher. This is an open access article distributed under the terms of the Creative Commons Attribution (CC BY-NC) license \\ (https://creativecommons.org/licenses/by-nc/4.0/). See http://ivyspring.com/terms for full terms and conditions.
}

Received: 2018.02.26; Accepted: 2018.05.25; Published: 2018.06.14

\begin{abstract}
Background: Oxidative stress plays pivotal roles in the progression of lung adenocarcinoma (LUAD) through cell signaling related closely to cancer growth. We previously reported that peroxiredoxin 4 (PRDX4), a secretory-type antioxidant enzyme, can protect against the development of various diseases, including potential malignancies. Since many patients with early-stage LUAD develop recurrence, even after curative complete resection, we investigated the association of the PRDX4 expression with the clinicopathological features and recurrence/prognosis using post-surgical samples of stage I-LUAD.
\end{abstract}

Methods: The expression of PRDX4 and MIB-1, a widely accepted Ki67 protein, was immunohistochemically analysed in 206 paraffin-embedded tumour specimens of patients with stage I-LUAD. The PRDX4 expression was considered to be weak when less than $25 \%$ of the adenocarcinoma cells showed positive staining.

Results: A weak PRDX4+ expression demonstrated a significantly close relationship with pathologically poor differentiation, highly invasive characteristics and recurrence. The decrease in PRDX4-positivity potentially induced cell growth in LUAD, which was correlated significantly with a very high MIB-1 labelling index $(\geq 17.3 \%)$. Univariate/multivariate analyses revealed that the subjects with both weak PRDX4+ expression and a very high MIB-1 index had significantly worse disease-free survival rates than other subjects.

Conclusions: The combination of weak PRDX4 expression and a very high MIB-1 index can predict high proliferating activity and recurrence with a potential poor prognosis, especially in post-operative stage I-LUAD patients.

Key words: lung adenocarcinoma (LUAD); stage I; PRDX4; MIB-1; recurrence.

\section{Introduction}

Lung cancer is one of the most common fatal malignancies in developed countries [1,2] and it has been the number-one cause of cancer-related deaths among Japanese for two decades. Up to 105,000 new cases of lung cancer were diagnosed in 2013, and in 2016, more than 50,000 patients died of it in Japan alone (http://ganjoho.jp/reg_stat/statistics/stat/ summary.html). More than $85 \%$ of lung cancer cases are classified as non-small cell lung cancer (NSCLC), and lung adenocarcinoma (LUAD) is the most well-known histopathological subtype of NSCLC in Japan [3]. The 5-year overall survival rate is 
reportedly less than $20 \%$ for NSCLC, including LUAD [4], and surprisingly, up to $30 \%$ of patients develop recurrence within 5 years, even in cases of stage I-LUAD after curative complete surgical resection $[5,6]$. The potential cell growth of LUAD, regardless occult metastases at the time of operation, is suggested to be primarily responsible for its recurrence with a subsequent poor prognosis [7]. Therefore, predicting which patients are prone to develop recurrence after surgery is critical, even with early-stage LUAD. Indeed, clinicopathological elements, such as the differentiation or tumour-node-metastasis (TNM) stage of LUAD, can strongly suggest the risk of recurrence and/or the prognosis $[8,9]$, but no molecular or genetic factors have yet been identified, and the clinical significance of such biological markers is still under evaluation.

Oxidative stress, induced by reactive oxygen species (ROS), can function as a crucial and diverse pathophysiological regulator of cellular signalling pathways, such as the response to inflammatory and growth factor stimulation [10]. Accumulating evidence also suggests that the dysregulation of oxidant and antioxidant redox signalling might cause or accelerate a host of various human diseases, including malignancies [11]. In this vein, the aberrant expressions of oxidative stressors and antioxidant properties play pivotal roles in the initiation of the progression of LUAD through cell signalling pathways related closely to cancer growth [12].

Peroxiredoxin 4 (PRDX4) is a member of the PRDX antioxidant enzyme family, which consists of at least six distinct PRDX genes, expressed in mammals (PRDX1-6) [13]. In contrast to the merely intracellular localization of other family members, PRDX4 is the only secretory form, and significant levels of this enzyme have been noted, particularly in cultured medium [14]. According to our serial in vivo studies, the elevated expression of PRDX4 has been recognized in not only endoplasmic reticulum but serum and various tissues of mice and human with chronic inflammatory diseases, manifesting as metabolic syndrome and potential malignancies $[15,16]$. The overexpression of PRDX4 in mice can markedly suppress the local and systemic levels of ROS and protect various tissues against oxidative damage by reducing the inflammatory response and apoptosis and/or growth factor stimulation in the intra-/extra-cellular space [17]. Furthermore, a growing body of evidence suggests that apoptotic and/or proliferative activities might be significantly correlated with the PRDX4 expression $[18,19]$.

Given the above, we hypothesize that PRDX4 not only regulates basic cellular functions of LUAD but is a parameter of cell growth, similar to the widely-accepted Ki67 (MIB-1) protein [20,21]. Furthermore, PRDX4 might be a promising clinical biomarker for the recurrence/prognosis of LUAD and be a target for early diagnoses and therapies for LUAD. However, no studies have explored possible associations between the PRDX4 expression, especially in early-stage LUAD, and the clinicopathological characteristics of a lesion, including its differentiation and invasiveness or patients' recurrence/prognosis.

In the current study, using an original, specific rabbit polyclonal PRDX4 antibody generated against the recombinant PRDX4 protein [22], we evaluated the expression of PRDX4 in post-surgical specimens using stage I-LUAD patients' clinicopathological data, demonstrating that PRDX4 was weakly expressed in most invasive human LUAD specimens, especially those with poor differentiation, pleural involvement, recurrence, and an MIB-1 labelling index exceeding $17.3 \%$ (i.e. very high proliferating activity). These findings suggest that the combination of weak PRDX4+ expression and a very high MIB-1 index is significantly correlated with a poor disease-free survival (DFS; i.e. recurrence) of stage I-LUAD.

\section{Materials and methods}

\section{Patients and tissue specimens}

Surgically resected stage I-LUAD tissues were evaluated in the present study. Pathological reports were reviewed to identify patients who underwent lobectomy (170 patients), partial resection (4 patients), or segmentectomy (32 patients) for LUAD between January 2005 and December 2015 at the hospital of Kanazawa Medical University. All materials in this article were approved by the Ethical Committee of Kanazawa Medical University (I159). Patients who suffered perioperative deaths, defined as death during the patient's initial hospitalization or within 30 days of surgery, were excluded. A total of 206 patients with available follow-up data comprised the cohort of this retrospective study after further excluding those with the following characteristics: (a) other prior or concomitant malignant tumours, (b) coexisting medical problems of sufficient severity to shorten the life expectancy, and (c) adjuvant chemotherapies or radiotherapies prior to the surgery.

Three pathologists examined all resected specimens to confirm their histopathological features, including the differentiation. Revisions in the International System for Staging Lung Cancer was used for the final staging [23], and all lung adenocarcinomas were further classified based on the histological classification system from the International Association for the Study of Lung 
Cancer (IASLC)/American Thoracic Society (ATS)/ European Respiratory Society (ERS)/International Multidisciplinary Classification of Lung Adenocarcinoma [24].

In accordance with this IASLC/ATS/ERS classification system [24], adenocarcinoma in situ (AIS) cases were selected using haematoxylin and eosin (H\&E)-stained sections according to the following criteria: localized lesion $(\leq 3 \mathrm{~cm})$ with growth of neoplastic cells along pre-existing alveolar structures, lack of stromal invasion, absence of papillary or micropapillary patterns, and absence of intra-alveolar tumour cells. Tumours were subclassified as minimally invasive adenocarcinoma (MIA) in cases with a solitary adenocarcinoma $(\leq 3 \mathrm{~cm})$ with a predominantly lepidic growth pattern and $\leq 5$ $\mathrm{mm}$ invasion in the greatest dimension of any one focus. The invasive component to be measured in MIA was defined as follows: histological subtypes other than a lepidic pattern (i.e. acinar, papillary, micropapillary, or solid) or tumour cells infiltrating myofibroblastic stroma. The invasive component was measured morphometrically, and a 5-mm cut-off value was used to distinguish MIA from lepidic-predominant invasive adenocarcinoma (LPA). For cases that contained multiple tumour foci, only the largest focus was examined. Elastica van Gieson (EVG) stains were also performed if necessary. MIA was excluded if the tumour invaded the lymphatics, blood vessels, pleura, or contained tumour necrosis. LPA and non-lepidic adenocarcinomas with invasion that were $>5 \mathrm{~mm}$ in diameter were classified as invasive adenocarcinoma and divided further into acinar (APA), papillary (PPA), solid (SPA), mucinous adenocarcinoma (MA), and micropapillary (MPA) based on their predominant invasive pattern in $\mathrm{H} \& \mathrm{E}$ sections.

Clinical information was gathered from patients' records. The disease-free survival (DFS) and disease-specific survival (DSS) were defined as the interval from the date of surgery to recurrence and from the date of surgery to death, except for patients who died from causes other than LUAD, or the most recent clinic visit, respectively. Patients were followed up and prospectively evaluated every month within the first postoperative year and at approximately twoto four-month intervals thereafter using chest X-ray, thoracic and abdominal computed tomography (CT), brain magnetic resonance imaging (MRI), serum biochemistry, or measurements of tumour markers. $\mathrm{CT}, \mathrm{MRI}$, and bone scintigraphy were performed every six months for three years after surgery. Additional examinations were performed if any symptoms or signs of recurrence were recognized. Formalin-fixed, paraffin-embedded tissue blocks came from our Department of Pathology \& laboratory medicine. EVG and immnohistochemical D2-40 (Nichirei Bioscience Co., Tokyo, Japan, diluted 1:1) staining very clearly revealed pleural involvement (pl) and vascular invasion (v) in the former, and lymphatic invasion (ly) in the latter, respectively.

\section{Preparation of antibodies against PRDX4 and secondary antibodies, and immunohistochemistry of tissue samples}

A rabbit anti-PRDX4 IgG was produced as previously described [22]. Immunohistochemical staining was performed by the antibody-linked dextran polymer method for antibody-bridge labelling, with haematoxylin counterstaining (EnVision; Dako Cytomation, Co., Glostrup, Denmark). Deparaffinized and rehydrated $4-\mu \mathrm{m}$ sections were incubated in $10 \% \mathrm{H}_{2} \mathrm{O}_{2}$ for 5 min to block the endogenous peroxidase activity. The sections were then rinsed and incubated with rabbit polyclonal anti-PRDX4 (diluted 1:1000) and mouse monoclonal MIB-1 (Ki67; Dako Cytomation, Co., diluted 1:50) antibodies for $2 \mathrm{~h}$ and $30 \mathrm{~min}$, respectively $[19,21]$. The second antibody-peroxidaselinked polymers were then applied, and the sections were incubated with a solution consisting of $20 \mathrm{mg}$ of $3.3^{\prime}$-diaminobenzidine tetrahydrochloride, $65 \mathrm{mg}$ of sodium azide, and $20 \mathrm{ml}$ of $30 \% \mathrm{H}_{2} \mathrm{O}_{2}$ in $100 \mathrm{ml}$ of Tris-HCL (50 mM, pH7.6). After counterstaining with Meyer's haematoxylin, the sections were observed under a light microscope. The sections were first scanned at a low power for all fields (original magnification: $\times 40$ ) with tumour and non-tumour tissues to account for the heterogeneity of distribution. The number of cells showing positive staining and the pattern of staining were recorded. Necrotic tissues, stromal cells, and lymphoid cells were not included in the recording.

\section{The evaluation of the immunohistochemical results by scoring}

The immunoreactivity for PRDX4 in each case was assessed semi-quantitatively by evaluating the proportion of positive cells compared to the total neoplastic LUAD cells. We selected and validated the immunohistochemical cut-off scores for PRDX4 positivity (25\%) and the MIB-1 labelling index (17.3\%) based on the performance of a receiver operating characteristic (ROC) curve analysis [25]. All patients were divided into two groups based on the PRDX4 expression as follows: strong when the PRDX4 staining was $\geq 25 \%$ and weak when the staining was $<25 \%$.

All histological and immunohistochemical slides were evaluated by two independent observers 
(certified surgical pathologists in our department; A.S. and N.K.) using a blind protocol design (observers blinded to the clinicopathological data). The agreement between the observers was excellent (more than 90\%) for all antibodies investigated, as measured by the interclass correlation coefficient. For the few (less than 1\%) instances of disagreement, a consensus score was determined by a third board-certified pathologist (S.Y.) in our department $[21,26,27]$.

Table 1. Detailed patients'clinicopathological characteristics

\begin{tabular}{|c|c|}
\hline Characteristic & Patients $(n=206)$ \\
\hline \multicolumn{2}{|l|}{ Age (years) } \\
\hline Average & 67 \\
\hline Median & 68 \\
\hline Range & $33-83$ \\
\hline$>60$ & 166 \\
\hline$\leq 60$ & 40 \\
\hline \multicolumn{2}{|l|}{ Sex } \\
\hline Male & 104 \\
\hline Female & 102 \\
\hline \multicolumn{2}{|c|}{ Brinkman index (BI) } \\
\hline$\geq 400$ & 78 \\
\hline$<400$ & 128 \\
\hline \multicolumn{2}{|c|}{ Months after surgery } \\
\hline Average & 51 \\
\hline Median & 49 \\
\hline Range & $2-145$ \\
\hline \multicolumn{2}{|c|}{ Tumour differentiation } \\
\hline Well & 112 \\
\hline Moderately & 78 \\
\hline Poorly & 16 \\
\hline \multicolumn{2}{|c|}{ Histopathological subtype } \\
\hline AIS & 19 \\
\hline MIA & 38 \\
\hline LPA & 52 \\
\hline APA & 29 \\
\hline PPA & 49 \\
\hline MA & 3 \\
\hline MPA & 3 \\
\hline SPA & 13 \\
\hline \multicolumn{2}{|c|}{ Tumour size (mm) } \\
\hline Average & 23.5 \\
\hline Median & 22 \\
\hline Range & $6-50$ \\
\hline \multicolumn{2}{|l|}{$\operatorname{CEA}(\mu \mathrm{g} / \mathrm{L})$} \\
\hline$\geq 5$ & 65 \\
\hline$<5$ & 141 \\
\hline
\end{tabular}

\section{Statistical analyses}

The significance of correlations was determined using Fisher's exact test or $\chi^{2}$ test, where appropriate, in order to assess the relationships between the immunohistochemical expression and the clinicopathological features [27]. Survival curves were plotted with the Kaplan-Meier method and compared with the log-rank test. Hazard ratios and 95\% confidence intervals (95\% CIs) were estimated using univariate or multivariate Cox proportional hazard models [21,26-29]. All statistical tests were two-tailed, with values of $\mathrm{P}<0.05$ considered to be significant.

All of the above statistical analyses were performed with the EZR (Saitama Medical Center, Jichi Medical University, Japan) graphical user interface for the $\mathrm{R}$ software program (The $\mathrm{R}$ Foundation for Statistical Computing, version 2.13.0) $[27,28,30]$. More precisely, it is a modified version of $R$ commander (version 1.6-3) that incorporates the statistical functions frequently used in biostatistics.

\section{Results}

\section{Patient characteristics}

The clinicopathological features of the 206 patients with stage I-LUAD who were able to be evaluated are summarized in Table 1 . The range of age at surgery was 33-83 years (average and median were 67 and 68 years, respectively). More than half of patients $(128 / 206)$ had a Brinkman index (BI) under 400 ; the remaining patients $(78 / 206)$ were $\geq 400 \mathrm{BI}$. The median tumour size was $22 \mathrm{~mm}$ (range: 6-50 $\mathrm{mm})$. The tumour grading included 112 welldifferentiated $(54.4 \%), 78$ moderately differentiated $(37.9 \%)$, and 16 poorly differentiated adenocarcinoma (7.8\%). According to further histopathological analyses with the IASLC/ATS/ERS classification system (Travis et al., 2011), 19 (9.2\%) patients had AIS, 38 (18.4\%) MIA, 52 (25.2\%) LPA, 29 (14.1\%) APA, 49 (23.8\%) PPA, 3 (1.5\%) MA, 3 (1.5\%) MPA, and 13 (6.3\%) SPA. Postoperative follow-up was available for all 206 patients (average: 51 months; range: 2-145 months). The median postoperative DFS was 42 months with a 1-year recurrence rate of 2.9\%, 2-year recurrence rate of $11.2 \%, 5$-year recurrence rate of $17 \%$, and total recurrence rate of $20 \%$.

\section{Association of the PRDX4 expression with the clinicopathological variables and DFS}

Based on the cut-off points for the PRDX4 and MIB-1 expression, all subjects were divided into two groups for each parameter: a weak and strong PRDX4 group and a low and high MIB-1 group (Figure 1). To clarify the association of PRDX4 expression (weak vs. strong PRDX4+) (Figure 2) with the clinicopathological characteristics of the cohort, the variables were split as shown in Table 2. There were no significant differences between the patients with weak and strong PRDX4+ tumour expressions in terms of the age, gender, and BI $(\mathrm{P}>0.05)$. The moderately to poorly differentiated tumour rate in the strong PRDX4+ samples was 30/103 (29.1\%), but the rate was 65/103 (63.1\%) in weak PRDX4+ samples. Furthermore, the highly invasive (APA/PPA/MA/ MPA/SPA) adenocarcinoma rate was 30/103 (29.1\%) 


\section{PRDX4 (vs. recurrence)}

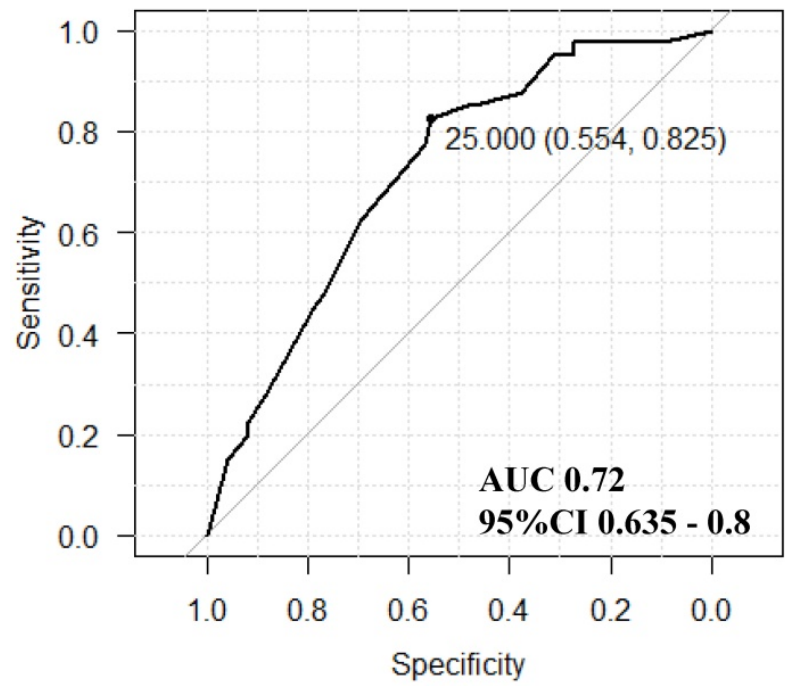

MIB-1 (vs. recurrence)

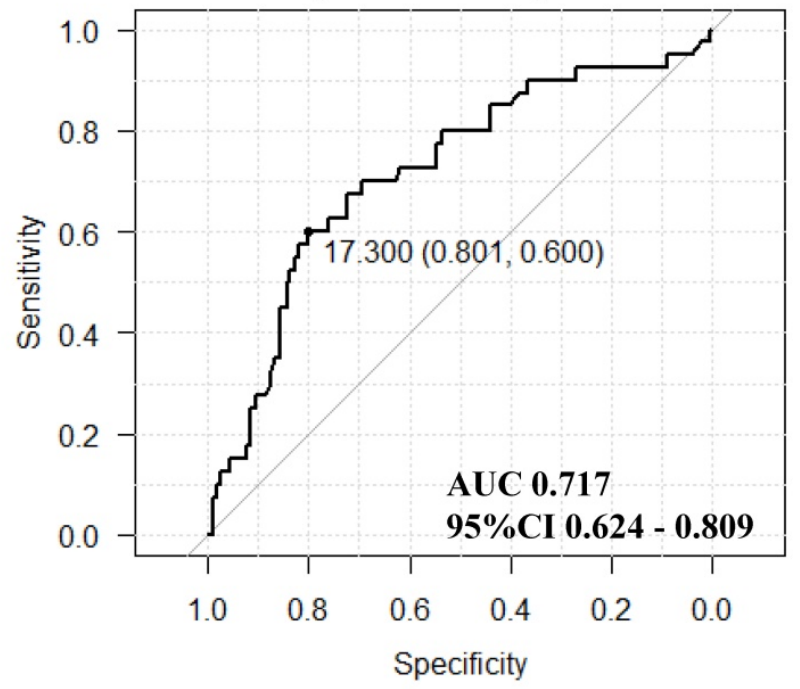

Figure 1. The results of the receiver operating characteristic (ROC) curve analyses for selecting and validating the immunohistochemical cut-off points for PRDX4 and MIB-1 expression. We selected the cut-off values of PRDX4 and MIB-1 using ROC and the area under the curve (AUC), as an effective measure of accuracy has been considered a meaningful interpretation. We selected 25 and 17.3, respectively, as the cut-off points for PRDX4 and MIB-1, since the AUC for recurrence was the highest among all clinicopathological variables.

in strong PRDX4+ samples but 67/103 (65\%) in weak PRDX4+ samples. Weak PRDX4 expression was closely associated with moderate to poor differentiation $(\mathrm{P}<0.0001)$, highly invasive subtypes (APA/PPA/MA/MPA/SPA) ( $<$ 0.0001), and a high $(\geq 17.3 \%)$ MIB-1 labelling index $(\mathrm{P}=0.0018, \mathrm{r}=-0.172)$ but not with the tumour size or presence of $\mathrm{v}$ and ly $(\mathrm{P}$ $>0.05)$. PRDX4 expression was apparently detectable in the adjacent non-neoplastic bronchioloalveolar epithelium (Figure 2). On immunohistochemistry, PRDX4 and MIB-1 displayed intracytoplasmic and nuclear expression patterns, respectively (Figure 2). Furthermore, the PRDX4 stain status was significantly correlated with the presence of $\mathrm{pl}(\mathrm{P}=0.017)$. The rate of PRDX4 expression in an intracytoplasmic pattern was much lower in invasive LUAD areas, including $\mathrm{pl}$ $(+)$, than in non-invasive ones (Figure 3 ).

In a Kaplan-Meier analysis, lung adenocarcinoma patients with weak PRDX4+ expression had a significantly shorter postoperative DFS than those with strong PRDX4+ expression $(\mathrm{P}=0.004$, Figure 4A). Lung adenocarcinoma patients with weak PRDX4+ and a high MIB-1 index had a markedly shorter postoperative DFS than other patients $(\mathrm{P}<$ 0.0001, Figure 4B). However, the PRDX4 expression was not associated with the postoperative DSS in the present study.

\section{The combination of weak PRDX4 expression and a high MIB-1 labelling index represents a significant independent prognostic indicator for lung adenocarcinoma}

To assess whether or not the PRDX4 expression was an independent predictor of postoperative DFS, a
Cox proportional-hazards model was created in a forward fashion including only covariates that had statistically significant correlations with the DFS, using an inclusion threshold of $\mathrm{P}<0.05$ (Table 3). A univariate analysis showed that the tumour size $(>2$ $\mathrm{cm})$, tumour grade, and presence of $\mathrm{pl}, \mathrm{ly}$, and $\mathrm{v}$ and both weak PRDX4+ and a high MIB-1 labelling index status, were significant predictors of a poor survival $(\mathrm{P}=0.021,<0.0001,<0.0001,=0.0002,<0.001$, and $<$ 0.0001, respectively). Furthermore, a multivariate analysis showed that, after correction for confounding variables, the combination of weak PRDX4+ expression and a high MIB-1 index remained an independent prognostic indicator for the DFS $(\mathrm{P}=$ $0.013)$, as well as the tumour grade $(\mathrm{P}=0.0009)$.

\section{Discussion}

In the present large cohort, we showed that weak PRDX4 expression was closely correlated with various critical clinicopathological features of 206 patients with post-surgical LUAD especially in stage I, using a unique polyclonal antibody raised against the distinctive, recombinant PRDX4 protein. The current findings have indicated, for the first time, that the combination of weak PRDX4+ expression and a very high MIB-1 labelling index is novel and powerful independent marker for post-operative recurrence with a potential poor outcome in stage I-LUAD patients. 

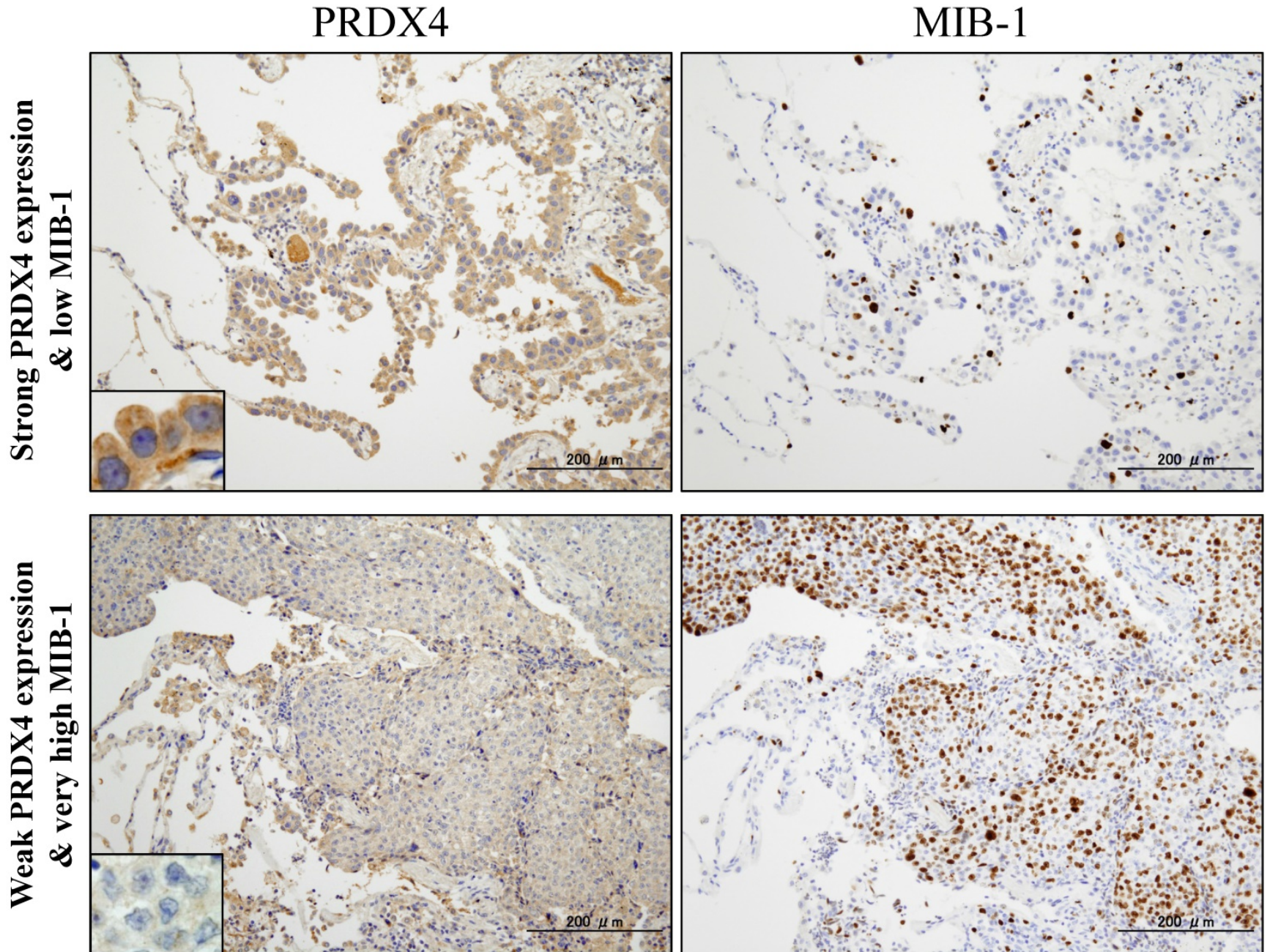

Figure 2. Representative images of immunohistochemical analyses of PRDX4 and MIB-1 in human stage I-LUAD (strong PRDX4 with low MIB-1; weak PRDX4 with high MIB-1). Intracytoplasmic staining pattern of PRDX4 was confirmed in LUAD cells (inset). (Original magnification: $\times 100 ;$ inset, $\times 400)$. Bar $=200 \mu \mathrm{m}(\times 100)$

Table 2. Detailed correlations between the PRDX4 expression and clinicopathological variables

\begin{tabular}{|c|c|c|c|}
\hline & $\begin{array}{l}\text { Strong expression } \\
(\mathrm{n}=103) \\
\text { Number }(\%)\end{array}$ & $\begin{array}{l}\text { Weak expression } \\
(\mathrm{n}=103) \\
\text { Number }(\%)\end{array}$ & $P$ \\
\hline \multicolumn{4}{|l|}{ Age } \\
\hline$>60$ years & $88(85.4)$ & $81(78.6)$ & 0.276 \\
\hline$\leq 60$ years & 15 (14.6) & $22(21.4)$ & \\
\hline \multicolumn{4}{|l|}{ Gender } \\
\hline Male & $46(44.7)$ & $58(56.3)$ & 0.125 \\
\hline Female & $57(55.3)$ & $45(43.7)$ & \\
\hline \multicolumn{4}{|c|}{ Brinkman index (BI) } \\
\hline$\geq 400$ & $36(35.0)$ & $45(43.7)$ & 0.254 \\
\hline$<400$ & $67(65.0)$ & $58(56.3)$ & \\
\hline \multicolumn{4}{|c|}{ Tumour differentiation } \\
\hline Well & $73(70.9)$ & $38(36.9)$ & $<0.0001$ \\
\hline Moderately & $25(24.3)$ & $54(52.4)$ & \\
\hline Poorly & $5(4.9)$ & $11(10.7)$ & \\
\hline \multicolumn{4}{|c|}{ Histopathological subtype } \\
\hline AIS & $13(12.6)$ & $6(5.8)$ & $<0.0001$ \\
\hline MIA & $28(27.2)$ & $10(9.7)$ & \\
\hline LPA & $32(31.1)$ & $20(19.4)$ & \\
\hline APA & $6(5.8)$ & $23(22.3)$ & \\
\hline PPA & $18(17.5)$ & $31(30.1)$ & \\
\hline MA & $1(1.0)$ & $2(1.9)$ & \\
\hline MPA & $2(1.9)$ & $1(1.0)$ & \\
\hline SPA & $3(2.9)$ & $10(9.7)$ & \\
\hline \multicolumn{4}{|c|}{ Tumour size } \\
\hline$>2 \mathrm{~cm}$ & $52(50.5)$ & $63(61.2)$ & 0.161 \\
\hline
\end{tabular}

\begin{tabular}{|c|c|c|c|}
\hline & $\begin{array}{l}\text { Strong expression } \\
(\mathrm{n}=103) \\
\text { Number }(\%)\end{array}$ & $\begin{array}{l}\text { Weak expression } \\
(\mathrm{n}=103) \\
\text { Number }(\%)\end{array}$ & $P$ \\
\hline$\leq 2 \mathrm{~cm}$ & $51(49.5)$ & $40(38.8)$ & \\
\hline \multicolumn{4}{|l|}{$\mathrm{pl}$} \\
\hline$(+)$ & $11(10.7)$ & $25(24.3)$ & \multirow[t]{2}{*}{0.017} \\
\hline$(-)$ & $92(89.3)$ & 78 (75.7) & \\
\hline \multicolumn{4}{|l|}{ ly } \\
\hline$(+)$ & $32(31.1)$ & $37(35.9)$ & \multirow[t]{2}{*}{0.555} \\
\hline$(-)$ & $71(68.9)$ & $66(64.1)$ & \\
\hline \multicolumn{4}{|l|}{$\mathbf{v}$} \\
\hline$(+)$ & $25(24.3)$ & $37(35.9)$ & \multirow[t]{2}{*}{0.095} \\
\hline$(-)$ & $78(75.7)$ & $66(64.1)$ & \\
\hline \multicolumn{4}{|l|}{ MIB-1 index } \\
\hline$\geq 17.3 \%$ (high) & $18(17.5)$ & $39(37.9)$ & \multirow[t]{2}{*}{0.0018} \\
\hline$<17.3 \%$ (low) & $85(82.5)$ & $64(62.1)$ & \\
\hline \multicolumn{4}{|l|}{ Recurrence } \\
\hline$(+)$ & $9(8.7)$ & $31(29.2)$ & \multirow[t]{2}{*}{0.0002} \\
\hline$(-)$ & $94(91.3)$ & $72(70.8)$ & \\
\hline \multicolumn{4}{|c|}{$\begin{array}{l}\text { AIS = adenocarcinoma in situ; MIA = minimally invasive adenocarcinoma; } \mathrm{LPA}= \\
\text { invasive adenocarcinoma, lepidic predominant; } \mathrm{APA} \text { = invasive adenocarcinoma, } \\
\text { acinar predominant; PPA = invasive adenocarcinoma, papillary predominant; SPA } \\
\text { = invasive adenocarcinoma, solid predominant; } \mathrm{MA} \text { = invasive mucinous } \\
\text { adenocarcinoma; } \mathrm{MPA}=\text { invasive adenocarcinoma, micropapillary predominant; } \\
\text { pl = pleural involvement; ly = lymphatic invasion; } \mathrm{v} \text { = vascular invasion. }\end{array}$} \\
\hline
\end{tabular}




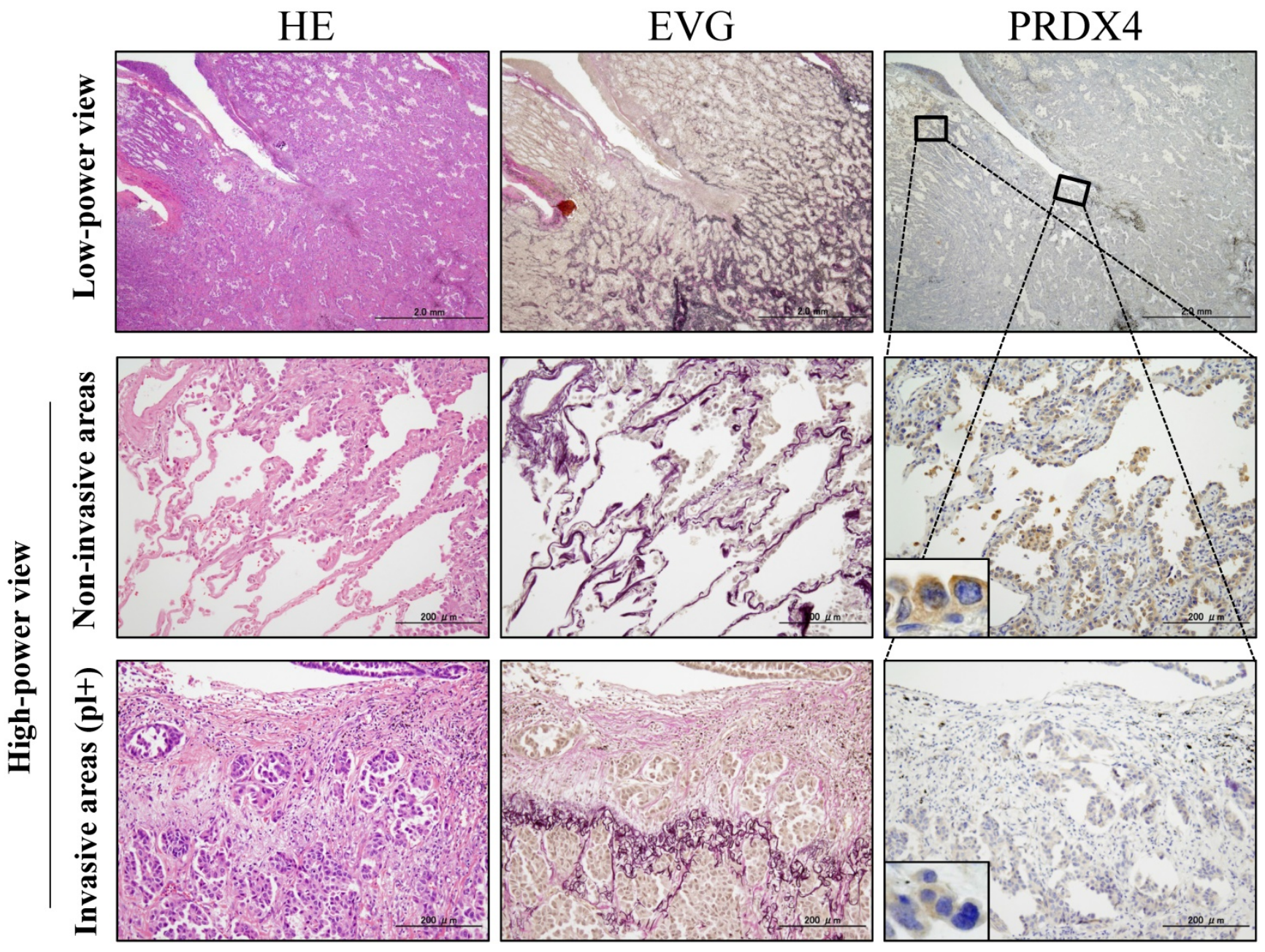

Figure 3. Representative pictures for H\&E, elastica van Gieson (EVG) and immunohistochemical analyses of PRDX4 in stage I-LUAD tissue with pleural involvement (Pl). EVG staining very clearly reveals elastic fibres of the visceral pleura $(\mathrm{Pl}(+))$. An intracytoplasmic staining pattern of PRDX4 was confirmed in LUAD cells (inset). (Original magnification: $\operatorname{Bar}=2 \mathrm{~mm}(\times 12.5)$ or $200 \mu \mathrm{m}(\times 100)$; inset, $\times 400)$.

A

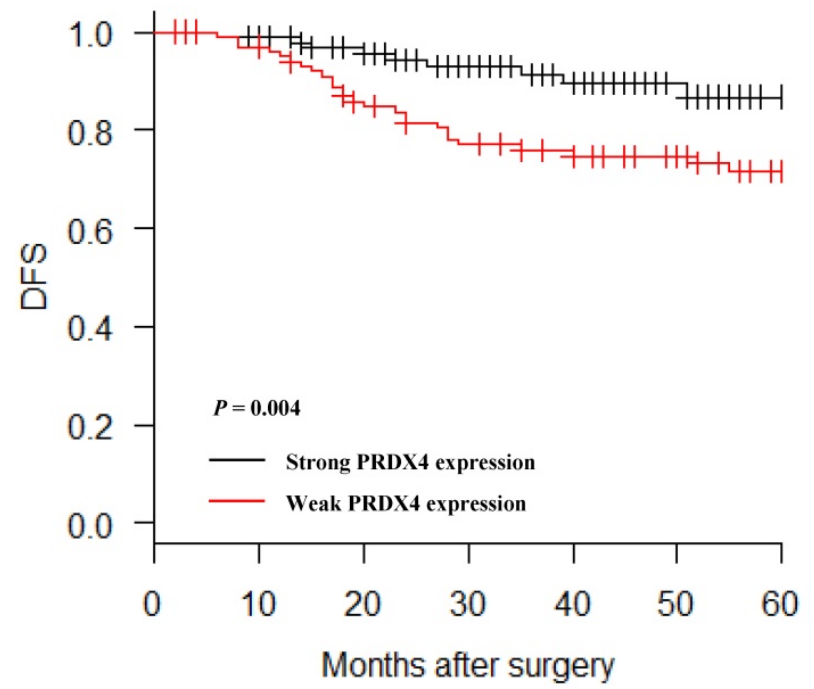

B

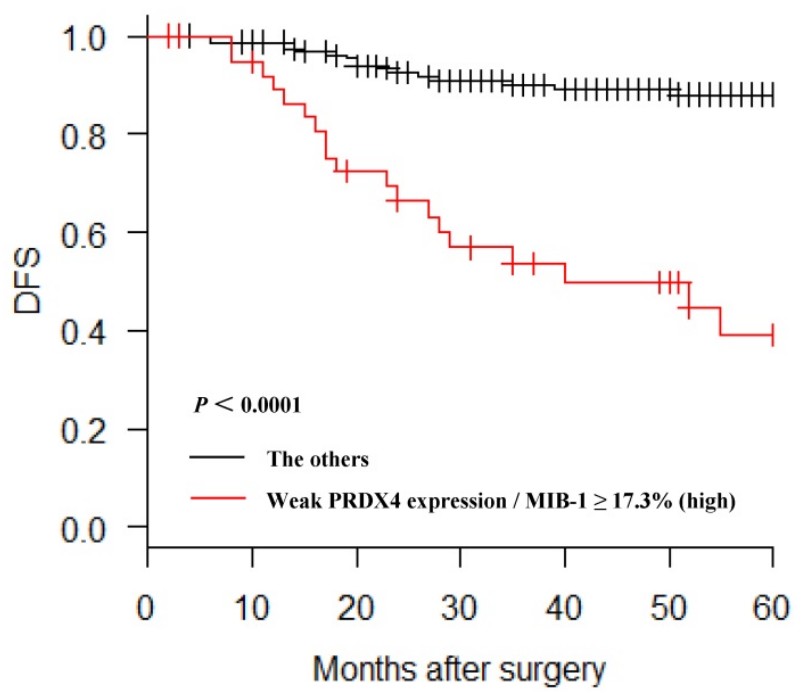

Figure 4. Kaplan-Meier curves of the disease-free survival (DFS) in patients with lung adenocarcinoma after surgery according to the PRDX4 expression. Weak PRDX4 expression alone as well as weak PRDX4/high MIB-1 is associated with a significantly shorter postsurgical DFS in stage I-LUAD patients. 
Table 3. Univariate and multivariate analyses of the survival in 206 patients with stage I-LUAD, according to the clinicopathological variables and a low PRDX4 expression and high MIB-1 labelling index

\begin{tabular}{|c|c|c|c|c|c|c|}
\hline & Univariate & & & Multivariate & & \\
\hline & Hazard ratio & $95 \% \mathrm{CI}$ & $P$-value & Hazard ratio & $95 \% \mathrm{CI}$ & $P$-value \\
\hline Weak PRDX4+high MIB-1 & 6 & $3.16-11.39$ & $<0.0001$ & 2.56 & $1.21-5.42$ & 0.013 \\
\hline Tumour size & 2.33 & $1.14-4.78$ & 0.021 & 2.14 & $0.94-4.89$ & 0.068 \\
\hline Differentiation & 14.22 & $5.05-40.01$ & $<0.0001$ & 13.1 & $2.88-59.67$ & 0.0009 \\
\hline pl (+) & 4.6 & $2.43-8.73$ & $<0.0001$ & 1.45 & $0.70-2.99$ & 0.316 \\
\hline ly $(+)$ & 3.77 & $1.88-7.59$ & 0.0002 & 1.32 & $0.61-2.89$ & 0.478 \\
\hline$v(+)$ & 3.99 & $2.02-7.87$ & $<0.0001$ & 0.89 & $0.39-1.99$ & 0.769 \\
\hline
\end{tabular}

Recurrence in LUAD patients after curative surgery remains a significant problem and can significantly affect the clinical course and survival of these patients [6,31]. Accumulated data suggest that weak PRDX4+ expression in stage I-LUAD is closely associated with pathological poorly differentiated characteristics and further invasive/aggressive behaviours, including pleural involvement or recurrence; furthermore, lesions with a weak PRDX4+ expression often co-express a very high MIB-1 labelling index $(\geq 17.3 \%)$, resulting in potential cell growth (i.e. high proliferating activity) of even early-stage LUAD. We were able to prove a critical, key specific antioxidant molecule, PRDX4, which should be poorly differentiated, invasive/ proliferative and recurrent tumour markers or therapeutic targets especially for stage I-LUAD.

However, some limitations associated with the present study warrant mention. First, this is a cohort-based, retrospective study at a single institution, even though we conducted thorough control through the random selection of post-operative stage I-LUAD patients and adherence to strict exclusion criteria. Second, we only conducted immunohistochemical and not detailed molecular analyses. Further in-depth follow-up in much larger cohorts of stage I-LUAD patients, along with detailed molecular investigations using LUAD cell culture lines, will be required to confirm the intriguing correlation of weak PRDX4+ expression and very high Ki67 expression with recurrence and a subsequent poor survival in post-surgical stage I-LUAD patients. The mechanism underlying how PRDX4 is involved in cellular signalling pathways, including the response to growth factor stimulation, should also be examined in a future research article. However, despite these limitations, the PRDX4 and/or Ki67 expression patterns in both post-/pre-operative tissue and serum samples of LUAD may allow for improved patient selection of candidates for adjuvant/ neoadjuvant systemic therapy as well as the early prediction of the clinical post-operative course. In addition, since secretory-type PRDX4 can appear in body fluids, it might be a quantitative soluble, tumour-specific marker for LUAD.
We suspected that PRDX4 might have a significant function of inhibiting ROS-related carcinogenesis of LUAD as a tumour suppressor through oxidant and antioxidant redox signalling pathways associated with cancer growth. Some of our present findings are in line with those of previous studies of several other human malignancies. For example, acute promyelocytic leukemia showed significantly reduced PRDX4 expression along with the control of granulocyte colony-stimulating factor (i.e. growth factor) responses [32]. Furthermore, our unpublished data suggest that human hepatocellular carcinoma specimens with a low expression of PRDX4 tend to have a highly malignant phenotype with a poor overall survival. However, other findings of ours disagree with those of other groups with regard to PRDX4 immunohistochemistry in squamous cell carcinomas (SCCs) $[33,34]$. These authors found that patients with an increased PRDX4 expression, which was closely associated with greater progressive activity, had a significantly shorter post-operative DSS in cases of oral cavity SCC [33] and DFS in cases of early-stage lung SCC [34] than those with a low expression. These discrepancies may be due in part to not only the heterogeneity of malignancies but also the methodology of assessment in each study, such as the size of the cohort, differences in the antibodies used against each PRDX4, and the arbitrary or strict selection and validation of the immunohistochemical cut-off scores for PRDX4, which were occasionally not based on any ROC curve analyses. Further experiments are necessary to address methodology standardization for PRDX4 in clinical specimens after collecting and investigating a much larger number of surgical cases.

\section{Conclusion}

Our observations suggest that weak PRDX4+ expression in primary stage I-LUAD is very closely related to pathological phenotypes with a poor outcome, e.g. those with poor differentiation, highly invasive characteristics and recurrence, or a very high MIB-1 labelling index, reflecting a background of marked cancer cell growth/proliferation. Furthermore, the DFS of LUAD patients with both weak PRDX4+ and a very high MIB-1 index was 
significantly shorter than that of other patients. These analyses suggest for the first time that the combination of weak PRDX4 and high MIB-1 may be a novel and useful independent predictor of recurrence with a poor prognosis in patients with primary stage I-LUAD.

\section{Abbreviations}

LUAD, lung adenocarcinoma; PRDX4, peroxiredoxin 4; NSCLC, non-small cell lung cancer; TNM, tumour-node-metastasis; ROS, reactive oxygen species; EVG, Elastica van Gieson; AIS, adenocarcinoma in situ; MIA, minimally invasive adenocarcinoma; LPA, invasive adenocarcinoma, lepidic predominant; APA, invasive adenocarcinoma, acinar predominant; PPA, invasive adenocarcinoma, papillary predominant; SPA, invasive adenocarcinoma, solid predominant; $\mathrm{MA}$, invasive mucinous adenocarcinoma; MPA, invasive adenocarcinoma, micropapillary predominant; DFS, disease-free survival; DSS, disease-specific survival; ROC, receiver operating characteristic and SCC, squamous cell carcinoma.

\section{Declarations}

\section{Ethics approval}

All materials including consent to participate in this article were approved by the Ethical Committee of Kanazawa Medical University (I159).

\section{Consent for publication}

Written informed consent was obtained from the patient the patient and his family on admission for the publication of this case report and any accompanying images.

\section{Availability of data and materials}

The dataset supporting the findings and conclusions of this research is included within the article.

\section{Acknowledgments}

We would like to thank Yuka Hiramatsu, Mariko Nakano and Manabu Yamashita for their expert technical assistance.

\section{Funding}

This work was supported in part by Grants-in-Aid for Scientific Research 16K08750 to S.Y. and 25462202 to H.U.) from the Ministry of Education, Culture, Sports, Science and Technology, Tokyo, Japan; a grant from the MSD Life Science Foundation, Public Interest Incorporated Foundation, Japan (to S.Y.); and grants from National Natural Science Foundation of China (No. 81402490) (to X.G.), Natural
Science Foundation of Hebei Province (No. H2016206170) (to X.G.), and High level talent support project of Hebei Province (No. CG2015003011) (to X.G.).

\section{Competing Interests}

The authors have declared that no competing interest exists.

\section{References}

1. Mitsudomi T, Suda K, Yatabe Y. Surgery for NSCLC in the era of personalized medicine. Nat Rev Clin Oncol. 2013; 10: 235-244.

2. Uramoto H. Current Topics on Salvage Thoracic Surgery in Patients with Primary Lung Cancer. Ann Thorac Cardiovasc Surg. 2016; 22: 65-68.

3. Lemjabbar-Alaoui H, Hassan OU, Yang YW, Buchanan P. Lung cancer: Biology and treatment options. Biochim Biophys Acta. 2015; 1856: 189-210.

4. Jemal A, Siegel R, Xu J and Ward E. Cancer statistics, 2010. CA Cancer J Clin. 2010; 60: 277-300.

5. Asamura H, Goya T, Koshiishi Y, Sohara Y, Eguchi K, Mori M, Nakanishi Y, Tsuchiya R, Shimokata K, Inoue H, Nukiwa T, Miyaoka E; Japanese Joint Committee of Lung Cancer Registry. A Japanese Lung Cancer Registry study: prognosis of 13,010 resected lung cancers. J Thorac Oncol. 2008; 3: 46-52.

6. Uramoto H, Yamada S, Tanaka F. Angiogenesis of lung cancer utilizes existing blood vessels rather than developing new vessels using signals from carcinogenesis. Anticancer Res. 2013; 33: 1913-1916.

7. Yamashita T, Uramoto H, Onitsuka T, Ono K, Baba T, So T, So T, Takenoyama M, Hanagiri T, Oyama T, Yasumoto K. Association between lymphangiogenesis-/micrometastasis- and adhesion-related molecules in resected stage I NSCLC. Lung Cancer. 2010; 70: 320-328.

8. Spiro SG, Silvestri GA. One hundred years of lung cancer. Am J Respir Crit Care Med. 2005; 172: 523-529.

9. Ou SH, Zell JA. Validation study of the proposed IASLC staging revisions of the T4 and $M$ non-small cell lung cancer descriptors using data from 23,583 patients in the California Cancer Registry. J Thorac Oncol. 2008; 3: 216-227.

10. Finkel T. Signal transduction by reactive oxygen species. J Cell Biol. 2011; 194: 7-15.

11. Fruehauf JP, Meyskens FL Jr. Reactive oxygen species: a breath of life or death? Clin Cancer Res. 2007; 13:789-94.

12. Okumura N, Yoshida H, Kitagishi Y, Nishimura Y, Iseki S, Matsuda S. Against Lung Cancer Cells: To Be, or Not to Be, That Is the Problem. Lung Cancer Int. 2012: 659365.

13. Fujii J, Ikeda Y, Kurahashi T, Homma T. Physiological and pathological views of peroxiredoxin 4. Free Radic Biol Med. 2015; 83: 373-379.

14. Okado-Matsumoto A, Matsumoto A, Fujii J, Taniguchi N. Peroxiredoxin IV is a secretable protein with heparin-binding properties under reduced conditions. J Biochem. 2000; 127:493-501.

15. Guo $X$, Yamada $S$, Tanimoto A, Ding $Y$, Wang KY, Shimajiri $S$, Murata $Y$, Kimura S, Tasaki T, Nabeshima A, Watanabe T, Kohno K, Sasaguri Y. Overexpression of peroxiredoxin 4 attenuates atherosclerosis in apolipoprotein E knockout mice. Antioxid Redox Signal. 2012; 17: 1362-1375.

16. Nabeshima A, Yamada S, Guo X, Tanimoto A, Wang KY, Shimajiri S, Kimura S, Tasaki T, Noguchi H, Kitada S, Watanabe T, Fujii J, Kohno K, Sasaguri Y. Peroxiredoxin 4 protects against nonalcoholic steatohepatitis and type 2diabetes in a nongenetic mouse model. Antioxid Redox Signal. 2013; 19: 1983-1998.

17. Yamada S, Ding Y, Sasaguri Y. Peroxiredoxin 4: Critical roles in inflammatory diseases. J UOEH. 2012; 34: 27-39.

18. Ding Y, Yamada S, Wang KY, Shimajiri S, Guo X, Tanimoto A, Murata Y, Kitajima S, Watanabe T, Izumi H, Kohno K, Sasaguri Y. Overexpression of peroxiredoxin 4 protects against high-dose streptozotocin-induced diabetes by suppressing oxidative stress and cytokines in transgenic mice. Antioxid Redox Signal. 2010; 13: 1477-1490.

19. Nawata A, Noguchi H, Mazaki Y, Kurahashi T, Izumi H, Wang KY, Guo X, Uramoto H, Kohno K, Taniguchi H, Tanaka Y, Fujii J, Sasaguri Y, Tanimoto A, Nakayama T, Yamada S. Overexpression of peroxiredoxin 4 affects intestinal function in a dietary mouse model of nonalcoholic fatty liver disease. PLoS One. 2016; 11: e0152549.

20. Burger PC, Shibata T, Kleihues P. The use of the monoclonal antibody Ki-67 in the identification of proliferating cells: application to surgical neuropathology. Am J Surg Pathol. 1986; 10: 611-617.

21. Kawatsu Y, Kitada S, Uramoto H, Li Z Takeda T, Kimura T, Horie S, Tanaka F, Sasaguri Y, Izumi H, Kohno K, Yamada S. The combination of strong expression of ZNF143 and high MIB-1 labelling index independently predicts shorter disease-specific survival in lung adenocarcinoma. Br J Cancer. 2014; 110: 2583-2592.

22. Ito R, Takahashi M, Ihara H, Tsukamoto H, Fujii J, Ikeda Y. Measurement of peroxiredoxin- 4 serum levels in rat tissue and its use as a potential marker for hepatic disease. Mol Med Rep. 2012; 6: 379-384. 
23. Vallières E, Shepherd FA, Crowley J, Van Houtte P, Postmus PE, Carney D, Chansky K, Shaikh Z, Goldstraw P. International Association for the Study of Lung Cancer International Staging Committee and Participating Institutions. The IASLC Lung Cancer Staging Project: proposals regarding the relevance of TNM in the pathologic staging of small cell lung cancer in the forthcoming (seventh) edition of the TNM classification for lung cancer. J Thorac Oncol. 2009; 4: 1049-1059.

24. Travis WD, Brambilla E, Noguchi M, Nicholson AG, Geisinger KR, Yatabe $Y$, Beer DG, Powell CA, Riely GJ, Van Schil PE, Garg K, Austin JH, Asamura H, Rusch VW, Hirsch FR, Scagliotti G, Mitsudomi T, Huber RM, Ishikawa Y, Jett J, Sanchez-Cespedes M, Sculier JP, Takahashi T, Tsuboi M, Vansteenkiste J, Wistuba I, Yang PC, Aberle D, Brambilla C, Flieder D, Franklin W, Gazdar A, Gould M, Hasleton P, Henderson D, Johnson B, Johnson D, Kerr K, Kuriyama K, Lee JS, Miller VA, Petersen I, Roggli V, Rosell R, Saijo N, Thunnissen E, Tsao M, Yankelewitz D. International association for the study of lung cancer/american thoracic society/european respiratory society international multidisciplinary classification of lung adenocarcinoma. J Thorac Oncol. 2011; 6: 244-285.

25. Hanley JA. Receiver operating characteristic (ROC) methodology: the state of the art. Crit Rev Diagn Imaging. 1989; 29: 307-335.

26. Harada $Y$, Izumi H., Noguchi H., Kuma A., Kawatsu Y, Kimura T., Kitada S, Uramoto H., Wang K.Y., Sasaguri Y., Hijioka H., Miyawaki A., Oya R., Nakayama T., Kohno K. and Yamada S. Strong expression of polypeptide $\mathrm{N}$-acetylgalactosaminyltransferase 3 independently predicts shortened disease-free survival in patients with early stage oral squamous cell carcinoma. Tumour Biol. 2016; 37: 1357-1368.

27. Hiraki T., Yamada S., Higashi M., Hatanaka K., Yokoyama S., Kitazono I., Goto Y., Kirishima M., Batra S.K., Yonezawa S. and Tanimoto A. Immunohistochemical expression of mucin antigens in gallbladder adenocarcinoma: MUC1-positive and MUC2-negative expression is associated with vessel invasion and shortened survival. Histol. Histopathol. 2017; 32: 585-596.

28. Kitada S, Yamada S, Kuma A, Ouchi S, Tasaki T, Nabeshima A, Noguchi H, Wang KY, Shimajiri S, Nakano R, Izumi H, Kohno K, Matsumoto T, Sasaguri $\mathrm{Y}$. Polypeptide $\mathrm{N}$-acetylgalactosaminyl transferase 3 independently predicts high-grade tumours and poor prognosis in patients with renal cell carcinomas. Br J Cancer. 2013; 109: 472-481.

29. Honjo K, Hiraki T, Higashi M, Noguchi H, Nomoto M, Yoshimura T, Batra SK, Yonezawa S, Semba I, Nakamura N, Tanimoto A, Yamada S. Immunohistochemical expression profiles of mucin antigens in salivary gland mucoepidermoid carcinoma: MUC4- and MUC6-negative expression predicts a shortened survival in the early postoperative phase. Histol Hitopathol. 2018; 33: 201-213

30. Kanda Y. Investigation of the freely available easy-to-use software 'EZR' for medical statistics. Bone Marrow Transplant. 2013; 48: 452-458.

31. Motono N, Matsui T, Machida Y, Usuda K, Uramoto H. Prognostic significance of histologic subtype in pStage I lung adenocarcinoma. Med Oncol. 2017: 34:100.

32. Palande KK, Beekman R, van der Meeren LE, Beverloo HB, Valk PJ, Touw IP. The antioxidant protein peroxiredoxin 4 is epigenetically down regulated in acute promyelocytic leukemia. PLoS One. 2011; 6: e16340.

33. Chang KP, Yu JS, Chien KY, Lee CW, Liang Y, Liao CT, Yen TC, Lee LY, Huang LL, Liu SC, Chang YS, Chi LM. Identification of PRDX4 and P4HA2 as metastasis-associated proteins in oral cavity squamous cell carcinoma by comparative tissue proteomics of microdissected specimens using iTRAQ technology. J Proteome Res. 2011; 10: 4935-4947.

34. Hwang JA, Song JS, Yu DY, Kim HR, Park HJ, Park YS, Kim WS, Choi CM. Peroxiredoxin 4 as an independent prognostic marker for survival in patients with early-stage lung squamous cell carcinoma. Int J Clin Exp Pathol. 2015; 8: $6627-6635$. 